\title{
A Note on the Ising Model in High Dimensions
}

\author{
J. Bricmont, ${ }^{1, \star}$ H. Kesten, ${ }^{2, \star \star}$ J. L. Lebowitz ${ }^{3, \star \star \star}$ and R. H. Schonmann ${ }^{2, \star \star \star \star}$ \\ ${ }^{1}$ Institut de Physique Theorique, Universite Catholique de Louvain, B-1348 Louvain-la-Neuve, \\ Belgium \\ ${ }^{2}$ Department of Mathematics, White Hall, Cornell University, Ithaca, NY 14853, USA \\ 3 Department of Mathematics, Busch Campus, Rutgers University, New Brunswick, NJ 08903, USA
}

\begin{abstract}
We consider the $d$-dimensional Ising model with a nearest neighbor ferromagnetic interaction $J(d)=1 / 4 d$. We show that as $d \rightarrow \infty$ the + phase (and the - phase) approaches a product measure with density given by the mean field approximation. In particular the spontaneous magnetization converges to its mean field value. A similar result holds for the unique Gibbs measure of the system subject to an external field $h \neq 0$.
\end{abstract}

\section{Introduction}

There exists a variety of rigorously established relations between statistical mechanical models and their mean field approximations. For several models the following types of results have been proven:

I) The mean field critical temperature is an upper bound for the critical temperature of the model (see [F] and [G] for the Ising model and [Si] for the classical Heisenberg model).

II) Convergence of the free energy to its mean field value when the dimensionality goes to infinity and the interaction is properly normalized (see [T3] for the Ising model and [PT] for generalizations; this result was first obtained nonrigorously in $[\mathrm{Br}]$ ).

III) The mean field value of the magnetization is an upper bound for the magnetization of the model. In particular this implies the result in (I) above (see [T1] for the Ising model and for instance [Pe, N, Sl, TH, V] for generalizations).

IV) Convergence of the critical temperature to its mean field value when the

* Part of this work was done while this author was visiting Rutgers University, supported by NSF grant DMR-86-12369 and Princeton University, support by NSF grant PHY-85-15288-A01

$\star \star$ Partially supported by a NSF grant to Cornell University

$\star \star \star$ Partially supported by NSF grant DMR 86-12369

$\star \star \star \star$ Supported by the U.S. Army Research Office through the Mathematical Sciences Institute of Cornell and by a NSF grant to Cornell University. This author was supported by the NSF grant DMR86-12369 while visiting Rutgers University (when this work was started). On leave from São Paulo University 
dimensionality goes to infinity and the interaction is properly normalized (this result follows for Ising and more generally for classical Heisenberg models from (I) above and the methods introduced in [FSS]; see Theorem 3.1 in that paper and Theorem 1 in $[\mathrm{BF}])$.

While not directly related to our results in this paper, we should also recall that convergence to mean field free energy can also be obtained in the limit of long range weak interactions (Kac limit; see [LP] and Appendix C in [T2]) and the important equality in large dimensions of critical exponents with their mean field values (see for instance $[\mathrm{AF}]$ and references given there).

In this note we prove another such relation for the $d$-dimensional nearest neighbor Ising model. We show that if the interaction is properly normalized, then as $d \rightarrow \infty$, the spontaneous magnetization converges to the value predicted by the corresponding mean field model. We show also that in the + phase the correlations between different spins vanish in this limit, so that in a certain sense this Gibbs state converges to a product measure whose density is given by the mean field approximation. When the system is subjected to an external magnetic field $h \neq 0$ (or when $\beta$ is small) there is only one phase and we prove that it also becomes of product form as $d \rightarrow \infty$. But in this case the fact that the corresponding magnetization converges to its mean field value can be easily obtained from the convergence of the free energy as stated in (II) above (see Theorem V.9.3 in [E]), since the free energy is convex and the mean field free energy is differentiable in $h$ for $h \neq 0$ (or when $\beta$ is small). That the convergence of the free energy is in general not enough to prove the convergence of the spontaneous magnetization can be seen by considering a one dimensional model with a Kac type of potential. For instance let each spin interact with its first $N$ neighbors to the right and to the left with constant strength $1 / 2 N$. In this case the spontaneous magnetization is 0 for every $N$ and every temperature, but the free energy converges as $N \rightarrow \infty$ to its mean field value (see Appendix C in [T2]). This limiting mean field free energy is not differentiable in $h$ at $h=0$ for low temperatures, giving rise to a positive mean field spontaneous magnetization. To our knowledge it is in fact an open problem in the setting of Kac type of potentials, to find the general conditions for results analogous to ours to hold, when phase transitions are present. (Unfortunately there are statements in the literature in which it is wrongly claimed that the interchange of limits above can be made even when the mean field free energy is not differentiable).

On the other hand, there is one example of a Kac type of potential for which the analogue of the theorem below can be proved by the methods of this paper when $d \geqq 3$ (i.e., the + phase converges to a product measure with density given by the mean field approximation). This is the model (b) of Sect. II of [BF]: the spins at sites $x$ and $y$ interact with a strength

$$
J_{x y}(\gamma)=\gamma^{2}\left(-\Delta+\gamma^{2}\right)^{-1}(x, y),
$$

where $\Delta$ is the finite difference Laplacian operator on $\mathbb{Z}^{d}$ and the mean field limit corresponds to $\gamma \rightarrow 0$. We leave to the interested reader the work of adapting our methods to handle this model. This relies only on the fact that $J_{x y}(\gamma)$ is reflection positive and that suitable infrared bounds hold. 
Before stating precisely out result we must introduce some notation. Consider the lattice $\mathbb{Z}^{d}$ and to each site $x \in \mathbb{Z}^{d}$ attach a spin $\sigma(x)= \pm 1$. The formal Hamiltonian will be given by

$$
H_{d, h}(\sigma)=-\frac{1}{4 d} \sum_{\langle x, y\rangle} \sigma(x) \sigma(y)-h \sum_{z} \sigma(z) .
$$

The first sum above runs over all pairs of nearest neighbors, each pair being included only once. The normalization factor $1 / 4 d$ in the Hamiltonian comes from a factor $1 / 2 d$ which is the inverse of the coordination number of the lattice and a factor $1 / 2$ which simplifies the comparison of the Ising model with more general Potts models and percolation (see [K1, K2 and KS]). Observe that with this choice the gap between the contribution to the energy due to a pair of parallel and a pair of opposite spins is $1 / 2 d$. The presence of $d$ in the denominator is natural, since we know already from $[\mathrm{BF}]$ that with such a scaling the inverse critical temperature converges to a positive finite constant; for the normalization which we use, this constant is 2 .

Let $\mu_{\Lambda, \eta, d, \beta, h}$ be the Gibbs measure with boundary condition $\eta \in\{-1,+1\}^{\mathbb{Z}^{d}}$ outside the finite region $\Lambda \subset \mathbb{Z}^{d}$ at inverse temperature $\beta$, i.e.,

$$
\mu_{\Lambda, \eta, d, \beta, h}(\sigma)=\left\{\begin{array}{l}
\exp \left(-\beta H_{\Lambda, d, h}(\sigma)-\beta W_{\Lambda, \eta, d}(\sigma)\right) \\
\text { if } \sigma(x)=\eta(x) \text { for every } x \in \Lambda^{\mathrm{c}} \\
0 \quad \text { otherwise. }
\end{array}\right.
$$

Here

$$
H_{\Lambda, d, h}(\sigma)=-\frac{1}{4 d} \sum_{\substack{\langle x, y\rangle \\ x, y \in \Lambda}} \sigma(x) \sigma(y)-h \sum_{z \in \Lambda} \sigma(z)
$$

and

$$
W_{\Lambda, \eta, d}(\sigma)=-\frac{1}{4 d} \sum_{\substack{\langle x, y\rangle \\ x \in \Lambda \\ y \notin \Lambda}} \sigma(x) \eta(y)
$$

It is well known [LM] (see also [Pr]) that if $h \neq 0$ then for every boundary condition $\eta, \mu_{\Lambda, \eta, d, \beta, h}$ converges weakly to a measure $\mu_{d, \beta, h}$ (which does not depend on $\eta$ ) as $\Lambda \uparrow \mathbb{Z}^{d}$ (in the sense that every finite region is eventually covered). If $\eta$ is the configuration identically +1 (which we will, as usual abbreviate by $\eta=+$ ), then for $h=0, \mu_{\Lambda,+, d, \beta, 0}$ converges weakly to the + phase $\mu_{+, d, \beta}$ as $\Lambda \uparrow \mathbb{Z}^{d}$.

We will use the notation $\langle\cdot\rangle$ for expected values with respect to Gibbs measures; we attach the same indices to the expectation $\langle\cdot\rangle$ as to the Gibbs measures. The spontaneous magnetization is defined by

$$
m(d, \beta):=\lim _{h \downarrow 0}\langle\sigma(0)\rangle_{d, \beta, h}=\langle\sigma(0)\rangle_{+, d, \beta}
$$

(see $[\mathrm{LM}]$ for the second equality), and the critical inverse temperature by

$$
\beta_{c}(d):=\inf \{\beta \geqq 0: m(d, \beta)>0\} .
$$

Later on it will also be convenient to consider the probability that a given spin is 
up in the + phase:

$$
\rho(d, \beta):=\mu_{+, d, \beta}(\sigma(0)=+1)=\frac{1+m(d, \beta)}{2} .
$$

The corresponding mean field model (see for instance Sect. V.9 of [E] or Sect. 4.5 of [T2] for details) is obtained by supposing that the spins outside the origin of $\mathbb{Z}^{d}$ assume the value given by the expectation of the spin at the origin. Denoting by $m(\beta, h)$ the expected value of the spin at the origin (in external field $h$ ) we write then the self conistency equation

$$
m(\beta, h)=\tanh ((\beta / 2)(m(\beta, h)+2 h)) .
$$

For $\beta \leqq 2$ or large $|h|$ this equation has only one solution, which is 0 when $h=0$, while for $\beta>2$ it has three solutions when $|h|$ is small. We restrict ourselves to the cases $h \geqq 0$ and denote by $m_{M F}(\beta, h)$ the largest solution (see the references given above for the motivation behind this definition). We will concentrate mainly on the case $h=0$ and abbreviate $m_{M F}(\beta):=m_{M F}(\beta, 0)$. The corresponding probability that the spin is up is

$$
\rho_{M F}(\beta)=\frac{1+m_{M F}(\beta)}{2} .
$$

More generally we will omit $h$ when it is 0 .

We will use also the following notation: $|\Gamma|$ will denote the cardinality of a set $\Gamma \subset \mathbb{Z}^{d} . \mathscr{P}(d)$ will denote the set of finite subsets of $\mathbb{Z}^{d}$. Given $\Gamma \in \mathscr{P}(d)$ we write $A(\Gamma)$ (respectively $B(\Gamma)$ ) for the event that all the spins in the region $\Gamma$ are +1 (respectively -1 ). Our main result can be stated as follows

Theorem. If $\beta \leqq 2$ then $m(d, \beta)=0$ for every $d$. For every $\beta \geqq 0$

$$
\begin{aligned}
& \lim _{d \rightarrow \infty} m(d, \beta)=m_{M F}(\beta), \\
& \lim _{d \rightarrow \infty} \rho(d, \beta)=\rho_{M F}(\beta),
\end{aligned}
$$

and more generally

$$
\lim _{d \rightarrow \infty} \sup _{\Gamma_{-}, \Gamma_{+} \in \mathscr{P}(d)}\left|\mu_{+, d, \beta}\left(A\left(\Gamma_{+}\right) \cap B\left(\Gamma_{-}\right)\right)-\left(\rho_{M F}(\beta)\right)^{\left|\Gamma_{+}\right|}\left(1-\rho_{M F}(\beta)\right)^{\mid \Gamma-1}\right|=0 .
$$

Remarks. 1) One would like to interpret this theorem as saying that the + phase converges weakly to a product measure. The trouble with such a statement is the fact that the lattice itself is changing with $d$. Still one can imbed each $\mathbb{Z}^{d}$ in the set $\left\{\left(x_{1}, x_{2}, \ldots\right): x_{i} \in \mathbb{Z}, i=1, \ldots\right.$ and for some $K, x_{j}=0$ if $\left.j \geqq K\right\}$ and give this statement a precise meaning.

2. As mentioned in the introduction, similar results can be obtained when $h \neq 0$ for the unique Gibbs measure $\mu_{d, \beta, h}$. We will address this point after the proof of the Theorem is completed.

3. Similar results were proven for an interacting particle system - the contact process - in [SV]. Using some of the methods in the present paper, they have also been obtained for ordinary percolation and some dependent percolation models [K1, K2]. Finally in [KS] such results were established for classical Heisenberg and Potts models using again in part methods employed here. 


\section{Proofs}

In the lemma below, $\langle f ; g\rangle:=\langle f g\rangle-\langle f\rangle\langle g\rangle$ and $\|\cdot\|$ stands for the Euclidean norm on $\mathbb{Z}^{d}$.

Lemma. For every $\beta \geqq 0$,

$$
\lim _{d \rightarrow \infty}\left(\frac{1}{2 d}\right)^{2} \sum_{\substack{\|x\|=1 \\\|y\|=1}}\langle\sigma(x) ; \sigma(y)\rangle_{+, d, \beta}=0 .
$$

Proof. The proof is based on the use of infrared bounds [FSS]. For every $d, \beta$ and $h \neq 0$ recall that $\mu_{d, \beta, h}$ is the unique infinite volume Gibbs measure. Let the measure $\omega_{d, \beta, h}$ on $R_{d}:=[-\pi, \pi]^{d}$ be such that

$$
\langle\sigma(0) \sigma(x)\rangle_{d, \beta, h}=\int_{R_{d}} e^{i k x} d \omega_{d, \beta, h}(k) .
$$

From [FSS] (see the proof of Theorem 3.1 there and remember that the external field only affects the a priori measure)

$$
d \omega_{d, \beta, h}(k)=(D(d, \beta, h) \delta(k)+g(d, \beta, h, k)) d^{d} k,
$$

where $D(d, \beta, h)$ is a constant, $\delta$ is the point mass concentrated on 0 and $g(d, \beta, h, k)$ is a non-negative integrable function satisfying

$$
g(d, \beta, h, k) \leqq \frac{1}{(2 \pi)^{d}} \frac{2 d \beta^{-1}}{\left(\sum_{i=1}^{d}\left(1-\cos k_{i}\right)\right)}
$$

From the triviality of the tail $\sigma$-field of $\mu_{d, \beta, h}$ (see Theorem 1.11 in [R]), translation invariance and the Riemann-Lebesgue Lemma [FSS] it follows now that

$$
\left(\langle\sigma(0)\rangle_{d, \beta, h}\right)^{2}=\lim _{\|x\| \rightarrow \infty}\langle\sigma(0) \sigma(x)\rangle_{d, \beta, h}=D(d, \beta, h) .
$$

From (2.2), (2.3) and translation invariance we obtain

$$
\begin{aligned}
\sum_{\substack{\|x\|=1 \\
\|y\|=1}}\left(\langle\sigma(x) \sigma(y)\rangle_{d, \beta, h}-D(d, \beta, h)\right) & =\sum_{\substack{\|x\|=1 \\
\|y\|=1}} \int_{R_{d}} e^{i k(x-y)} g(k) d^{d} k \\
& =\int_{R_{d}}\left(2 \sum_{i=1}^{d} \cos k_{i}\right)^{2} g(k) d^{d} k .
\end{aligned}
$$

Since the integrand in the right-hand side of (2.6) is positive, it follows from (2.4) and (2.6) that

$$
\begin{gathered}
\left(\frac{1}{2 d}\right)^{2} \sum_{\substack{\|x\|=1 \\
\|y\|=1}}\left\{\langle\sigma(x) \sigma(y)\rangle_{d, \beta, h}-D(d, \beta, h)\right\} \\
\leqq \frac{2}{\beta}\left(\frac{1}{2 \pi}\right)^{d} \int_{\boldsymbol{R}_{d}} \frac{\left(\frac{1}{d} \sum \cos k_{i}\right)^{2}}{1-\frac{1}{d} \sum_{i=1}^{d} \cos k_{i}} d^{d} k=: \frac{2}{\beta} I_{d} .
\end{gathered}
$$

As $h \downarrow 0, \mu_{d, \beta, h}$ converges weakly to $\mu_{+, d, \beta}$ (see Lemma 1 in [LM]). In particular, 
using (2.5)

$$
D(d, \beta, h)=\left(\langle\sigma(0)\rangle_{d, \beta, h}\right)^{2} \rightarrow(m(d, \beta))^{2}
$$

and

$$
\langle\sigma(x) \sigma(y)\rangle_{d, \beta, h} \rightarrow\langle\sigma(x) \sigma(y)\rangle_{+, d, \beta} .
$$

Therefore (2.8) implies, by taking the limit $h \downarrow 0$, that

$$
\left(\frac{1}{2 d}\right)^{2} \sum_{\substack{\|x\|=1 \\\|y\|=1}}\left(\langle\sigma(x) \sigma(y)\rangle_{+, d, \beta}-(m(d, \beta))^{2}\right) \leqq \frac{2}{\beta} I_{d} .
$$

Finally, we prove $I_{d} \rightarrow 0$, or more sharply,

$$
d I_{d} \rightarrow \frac{1}{2} \quad(d \rightarrow \infty)
$$

We owe the proof below to M. Aizenman; it is much simpler than our original proof. First write

$$
d I_{d}=2 E\left\{\left(\frac{1}{\sqrt{ } d} \sum_{1}^{d}\left[Z_{i}^{2}-E\left(Z_{i}^{2}\right)\right]\right)^{2}\left(\frac{1}{d} \sum_{1}^{d} Z_{i}^{2}\right)^{-1}\right\},
$$

where $E$ denotes expectation with respect to the probability measure $P(d k)=$ $(2 \pi)^{-d} d^{d} k$ on $R_{d}$, and $Z_{i}=\sin \left(k_{i} / 2\right)$. Here we used of course $1-\cos k_{i}=2 Z_{i}^{2}$ and $E\left(Z_{i}^{2}\right)=\frac{1}{2}$. Since the $Z_{i}^{2}$ are independent, identically distributed under the measure $P$, with $E\left(Z_{i}^{4}\right)=3 / 8$, we have by the central limit theorem that

$$
\frac{1}{\sqrt{ } d} \sum_{1}^{d}\left[Z_{i}^{2}-E\left(Z_{i}^{2}\right)\right] \rightarrow N(0,1 / 8) \text { in distribution, }
$$

where $N(0,1 / 8)$ is a normal variable with mean zero and variance $1 / 8$. Also, by the weak law of large numbers

$$
\frac{1}{d} \sum_{1}^{d} Z_{i}^{2} \rightarrow \frac{1}{2} \text { in distribution. }
$$

Standard theorems (e.g. [Bi], Corollary to Theorem (5.12)) therefore allow us to conclude from this that

$$
d I_{d} \rightarrow 2 \cdot \frac{1}{8}\left(\frac{1}{2}\right)^{-1}=\frac{1}{2},
$$

provided we can show that

$$
E\left\{\left|\frac{1}{\sqrt{ } d} \sum_{1}^{d}\left[Z_{i}^{2}-E\left(Z_{i}^{2}\right)\right]\right|^{3}\left(\frac{1}{d} \sum_{1}^{d} Z_{i}^{2}\right)^{-3 / 2}\right\}
$$

is bounded as $d \rightarrow \infty$. But by Hölder's inequality (2.12) is bounded by

$$
\left(E\left\{\left(\frac{1}{\sqrt{ } d} \sum_{1}^{d}\left[Z_{i}^{2}-E\left(Z_{i}^{2}\right)\right]\right)^{12}\right\}\right)^{1 / 4} \cdot\left(E\left\{\frac{1}{d} \sum_{1}^{d}\left(Z_{i}^{2}\right)^{-2}\right\}\right)^{3 / 4} .
$$

It is routine (compare [Bi], p. 409) to show that the first factor here is bounded, while the second factor can be handled by means of the harmonic-arithmetic mean inequality. Indeed. 
where

$$
\frac{d}{\sum_{1}^{d} Z_{i}^{2}} \leqq \frac{25}{d} \sum_{j} \frac{1}{V_{j}}
$$

$$
V_{j}=Z_{5 j+1}^{2}+\cdots+Z_{5 j+5}^{2} \text { for } 5 j+10<d,
$$

and for the single $j$ with $5 j+5<d \leqq 5 j+10$,

$$
V_{j}=Z_{5 j+1}^{2}+\cdots+Z_{d}^{2} \text {. }
$$

Since $V_{j}^{-2}$ is integrable when $d \geqq 5$, we see that

$$
\left(E\left\{\left(\frac{1}{d} \sum_{1}^{d} Z_{i}^{2}\right)^{-2}\right\}\right)^{1 / 2} \leqq \frac{25}{d}\left(E\left\{\left(\sum_{j} V_{j}^{-1}\right)^{2}\right\}\right)^{1 / 2} \leqq \frac{25}{d} \sum_{j}\left(E\left\{V_{j}^{-2}\right\}\right)^{1 / 2} .
$$

(2.1) follows from (2.10) and (2.11).

Remark. 4) An alternative proof of (2.10) can be obtained from results in [FILS] for the + phase.

Proof of the Theorem. As observed in the introduction, the first statement of the theorem was proven for the Ising model in [T1].

We will now prove (1.8) (which is clearly equivalent to (1.9)) in Step 1 below and then use it to prove (1.10).

Step 1. Conditioning on the random variables $\{\sigma(x):\|x\|=1\}$ and using the Markov property of Gibbs measures we obtain

$$
m(d, \beta)=\left\langle\tanh \left(\frac{\beta}{4 d} \sum_{\|x\|=1} \sigma(x)\right)\right\rangle_{+, d, \beta} .
$$

From the Lemma,

$$
\frac{1}{2 d} \sum_{\|x\|=1} \sigma(x)-m(d, \beta) \rightarrow 0
$$

in distribution with respect to $\mu_{+, d, \beta}$, as $d \rightarrow \infty$. To see this observe that by (2.1) the variance of the random variable in (2.14) tends to 0 as $d \rightarrow \infty$, while, by translation invariance, its expectation is 0 for every $d$. Let

$\left(d_{k}: k=1, \ldots\right)$ be a sequence such that for some $l$,

$$
\lim _{k \rightarrow \infty} m\left(d_{k}, \beta\right)=l \text {. }
$$

Then from (2.14),

$$
\frac{1}{2 d_{k}} \sum_{\|x\|=1} \sigma(x) \rightarrow l
$$

in distribution, as $k \rightarrow \infty$. Since $\tanh (\cdot)$ is bounded and continuous, (2.13) and (2.16) imply

$$
\lim _{k \rightarrow \infty} m\left(d_{k}, \beta\right)=\tanh ((\beta / 2) l) .
$$

Comparing (2.15) with (2.17) yields 


$$
l=\tanh ((\beta / 2) l) .
$$

In words, any limiting point of $m(d, \beta)$ must satisfy the mean field equation (2.18). In case $\beta \leqq 2$ this equation has the unique solution, $l=m_{M F}(\beta)=0$, and so $m(d, \beta)$ must converge to this value. But in this case we know already that $m(d, \beta)=0$ for every $d$. More interesting is the case $\beta>2$; then $m_{M F}(\beta)>0$ and (2.18) has three solutions: $l=-m_{M F}(\beta), l=0, l=m_{M F}(\beta)$. But we know also from infrared bounds (see the proof of Theorem 3.1 in [FSS] and the proof of Theorem 1 in [BF]) that

$$
\underset{d \rightarrow \infty}{\liminf } m(d, \beta) \geqq(1-2 / \beta)^{1 / 2}>0 .
$$

Thus $m(d, \beta)$ must converge to $m_{M F}(\beta)$ also in this case.

Step 2. We will prove that for every $N$,

$$
\lim _{d \rightarrow \infty} \sup _{\substack{\Gamma \in \mathscr{P}(d, N) \\ 0 \notin \Gamma}}\left|\mu_{+, d, \beta}(\sigma(0)=+1 \mid A(\Gamma))-\rho_{M F}(\beta)\right|=0
$$

where

$$
\mathscr{P}(d, N)=\left\{\Gamma \subset \mathbb{Z}^{d}:|\Gamma| \leqq N\right\}
$$

From (2.14) and (1.8),

$$
\frac{1}{2 d} \sum_{\|x\|=1} \sigma(x) \rightarrow m_{M F}(\beta)
$$

(with respect to $\mu_{+, d, \beta}$ ) in distribution as $d \rightarrow \infty$. Now, given $\delta>0$, consider the event

$$
E_{\delta}=\left\{\frac{1}{2 d} \sum_{\|x\|=1} \sigma(x) \in\left(m_{M F}-\delta, m_{M F}+\delta\right)\right\} .
$$

(Equation 2.21) can be rephased by saying that for every $\delta>0$,

$$
\lim _{d \rightarrow \infty} \mu_{+, d, \beta}\left(\left(E_{\delta}\right)^{c}\right)=0 .
$$

Observe also that if we define $\alpha=1 /\left(1+e^{\beta}\right)$, then

$$
\mu_{+, d, \beta}(A(\Gamma)) \geqq \alpha^{|\Gamma|} \text {. }
$$

Thus, if $|\Gamma| \leqq N$ then

$$
\mu_{+, d, \beta}\left(\left(E_{\delta}\right)^{c} \mid A(\Gamma)\right) \leqq \frac{\mu_{+, d, \beta}\left(\left(E_{\delta}\right)^{c}\right)}{\mu_{+, d, \beta}(A(\Gamma))} \leqq \alpha^{-N} \mu_{+, d, \beta}\left(\left(E_{\delta}\right)^{c}\right) .
$$

Write now

$$
\begin{aligned}
& \left|\mu_{+, d, \beta}(\sigma(0)=+1 \mid A(\Gamma))-\rho_{M F}(\beta)\right|=\mid \mu_{+, d, \beta}\left(\sigma(0)=+1 \mid A(\Gamma) \cap E_{\delta}\right) \cdot \mu_{+, d, \beta}\left(E_{\delta} \mid A(\Gamma)\right) \\
& \quad+\mu_{+, d, \beta}\left(\sigma(0)=+1 \mid A(\Gamma) \cap\left(E_{\delta}\right)^{c}\right) \cdot \mu_{+, d, \beta}\left(\left(E_{\delta}\right)^{c} \mid A(\Gamma)\right) \\
& \quad-\rho_{M F}(\beta) \cdot \mu_{+, d, \beta}\left(E_{\delta} \mid A(\Gamma)\right)-\rho_{M F}(\beta) \cdot \mu_{+, d, \beta}\left(\left(E_{\delta}\right)^{c} \mid A(\Gamma)\right) \mid \\
& \leqq \\
& \left|\mu_{+, d, \beta}\left(\sigma(0)=+1 \mid A(\Gamma) \cap E_{\delta}\right)-\rho_{M F}(\beta)\right|+\mu_{+, d, \beta}\left(\left(E_{\delta}\right)^{c} \mid A(\Gamma)\right) .
\end{aligned}
$$

Using (2.23)-(2.25) we obtain 


$$
\begin{aligned}
& \underset{d \rightarrow \infty}{\limsup } \sup _{\substack{\Gamma \in \mathscr{P}(d, N) \\
0 \notin \Gamma}}\left|\mu_{+, d, \beta}(\sigma(0)=+1 \mid A(\Gamma))-\rho_{M F}(\beta)\right| \\
& \leqq \limsup _{d \rightarrow \infty} \sup _{\substack{\Gamma \in \mathscr{P}(d, N) \\
0 \notin \Gamma}}\left|\mu_{+, d, \beta}\left(\sigma(0)=+1 \mid A(\Gamma) \cap E_{\delta}\right)-\rho_{M F}(\beta)\right| .
\end{aligned}
$$

But by the Markov property of the Gibbs measures,

$$
\mu_{+, d, \beta}\left(\sigma(0)=+1 \mid A(\Gamma) \cap E_{\delta}\right) \in\left\{g(m): m_{M F}-\delta \leqq m \leqq m_{M F}+\delta\right\},
$$

where

$$
g(m)=\frac{1}{1+\exp (-\beta m)} .
$$

Indeed, the left-hand side of (2.27) is a convex combination of $g(m)$ 's with $m_{M F}-\delta \leqq m \leqq m_{M F}+\delta$ (by the definition of $E_{\delta}$ ).

Since $\delta>0$ is arbitrary, (2.20) follows from (2.26)-(2.28), the continuity of $g(\cdot)$ and the fact that $g\left(m_{M F}(\beta)\right)=\rho_{M F}(\beta)$. The last identity can be easily verified from (1.6) and (1.7).

Step 3. It follows easily from (2.20) and translation invariance that for every $N$

$$
\lim _{d \rightarrow \infty} \sup _{\Gamma \in \mathscr{P}(d, N)}\left|\mu_{+, d, \beta}(A(\Gamma))-\left(\rho_{M F}(\beta, h)\right)^{|\Gamma|}\right|=0
$$

(Use induction on $N$ ).

By the inclusion-exclusion principle (using induction on $\left|\Gamma_{-}\right|$) we have then for every $N$

$$
\lim _{d \rightarrow \infty} \sup _{\Gamma_{-}, \Gamma_{+} \in \mathscr{P}(d, N)}\left|\mu_{+, d, \beta}\left(A\left(\Gamma_{+}\right) \cap B\left(\Gamma_{-}\right)\right)-\left(\rho_{M F}(\beta)\right)^{\left|\Gamma_{+}\right|}\left(1-\rho_{M F}(\beta)\right)^{|\Gamma-|}\right|=0 .
$$

Step 4. To finish the proof of (1.10) we have to enhance the uniformity in $(2.30)$ by removing the bound $N$ on the cardinality of $\Gamma_{-}$and $\Gamma_{+}$.

Given $\varepsilon>0$, take $N$ such that

$$
\left(\rho_{M F}(\beta)\right)^{N} \leqq \varepsilon .
$$

For each $d$ we can choose $\Gamma_{+}(N) \subset \Gamma_{+}$and $\Gamma_{-}(N) \subset \Gamma_{-}$such that $\left|\Gamma_{+}(N)\right| \leqq N$, $\left|\Gamma_{-}(N)\right| \leqq N$. By (2.30) we can find $d$ large enough so that uniformly in $\Gamma_{+}$and $\Gamma_{-}$,

$$
\begin{aligned}
\mu_{+, d, \beta}\left(A\left(\Gamma_{+}\right) \cap B\left(\Gamma_{-}\right)\right) & \leqq \mu_{+, d, \beta}\left(A\left(\Gamma_{+}(N)\right) \cap B\left(\Gamma_{-}(N)\right)\right. \\
& \leqq\left(\rho_{M F}(\beta)\right)^{\left|\Gamma_{+}(N)\right|}\left(1-\rho_{M F}(\beta)\right)^{\mid\left(\Gamma_{-}(N) \mid\right.}+\varepsilon .
\end{aligned}
$$

The difference between two positive numbers is not greater than the largest of them, hence by (2.32),

$$
\begin{aligned}
& \left|\mu_{+, d, \beta}\left(A\left(\Gamma_{+}\right) \cap B\left(\Gamma_{-}\right)\right)-\left(\rho_{M F}(\beta)\right)^{\left|\Gamma_{+}\right|}\left(1-\rho_{M F}(\beta)\right)^{\mid \Gamma-1}\right| \\
& \quad \leqq\left(\rho_{M F}(\beta)\right)^{\left|\Gamma_{+}(N)\right|}\left(1-\rho_{M F}(\beta)\right)^{|\Gamma-(N)|}+\varepsilon .
\end{aligned}
$$

But $m_{M F}(\beta) \geqq 0$ and so $1-\rho_{M F}(\beta) \leqq \rho_{M F}(\beta)$. Thus the right-hand side of (2.33) is bounded above by

$$
\left(\rho_{M F}(\beta)\right)^{\left|\Gamma_{+}(N)\right|+|\Gamma-(N)|}+\varepsilon
$$


If $\left|\Gamma_{+}\right|>N$ or $\left|\Gamma_{-}\right|>N$, we have now from (2.31) and (2.33) with a suitable choice of $\Gamma_{ \pm}(N)$,

$$
\left|\mu_{+, d, \beta}\left(A\left(\Gamma_{+}\right) \cap B\left(\Gamma_{-}\right)\right)-\left(\rho_{M F}(\beta)\right)^{\left|\Gamma_{+}\right|}\left(1-\rho_{M F}(\beta)\right)^{|\Gamma-|}\right| \leqq 2 \varepsilon .
$$

This completes the proof of (1.10).

Remark. 5) The analogue of (2.1) in case $h \neq 0$ follows from (2.7) and (2.11). One can therefore prove the analogue of (1.10) when $h \neq 0$ by the same arguments as used above.

Acknowledgement. We would like to thank M. Aizenman for discussions.

\section{References}

[AF] Aizenman, M., Fernandez, R.: On the critical behavior of the magnetization in highdimensional Ising models. J. Stat. Phys. 44, 395-454 (1986)

[Bi] Billingsley, P.: Probability and measure, $2^{\text {nd }}$ ed. New York: John Wiley 1986

[Br] Brout, R.: Statistical mechanical theory of ferromagnetism high density behavior. Phys. Rev. 118, 1009-1019 (1960)

[BF] Bricmont, J., Fontaine, J. R.: Perturbation about the mean field critical point. Commun. Math. Phys. 86, 337-362 (1982)

[E] Ellis, R.: Entropy, large deviations and statistical mechanics. Berlin, Heidelberg, New York: Springer 1985

[F] Fisher, M.: Critical temperatures of anisotropic Ising lattices. II. General upper bounds. Phys. Rev. 162, 480-485 (1967)

[FILS] Fröhlich, J., Israel, R., Lieb, E. H., Simon, B.: Phase transition and reflexion positivity. I. General theory and long range lattice models. Commun. Math. Phys. 62, 1-34 (1978)

[FSS] Fröhlich, J., Simon, B., Spencer, T.: Infrared bounds, phase transitions and continuous symmetry breaking. Commun. Math. Phys. 50, 79-85 (1976)

[G] Griffiths, R. B.: Correlations in Ising ferromagnets. III. Commun. Math. Phys. 6, 121-127 (1967)

[K1] Kesten, H.: Asymptotics in high dimension for percolation. To appear in Festschrift in honor of J. M. Hammersley

[K2] Kesten, H.: Asymptotics in high dimension for the Fortuin-Kasteleyn random cluster model. To appear in Festschrift in honor of T. E. Harris

[KS] Kesten, H., Schonmann, R. H.: Behavior in large dimensions of the Potts and Heisenberg models. To appear in Rev. Math. Phys.

[LM] Lebowitz, J. L., Martin-Löf, A.: On the uniqueness of the equilibrium state for Ising spin systems. Commun. Math. Phys. 25, 276-282 (1972)

[LP] Lebowitz, J. L., Penrose, O.: Rigorous treatment of the van der Waals-Maxwell theory of liquid-vapor transition. J. Math. Phys. 7, 98-113 (1966)

[N] Newman, C. M.: Shock waves and mean field bounds. Concavity and analyticity of the magnetization at low temperatures. Appendix to Percolation theory: A selective survey of rigorous results, pp.147-167. In Advances in multiphase flow and related problems. Papanicolaou, G., (ed.): SIAM 1987

[Pe] Pearce, P. A.: Mean field bounds on the magnetization for ferromagnetic spin models. J. Stat. Phys. 25, 309-320 (1981)

[Pr] Preston, C. J.: An application of the GHS inequalities to show the absence of phase transition for Ising spin systems. Commun. Math. Phys. 35, 253-255 (1974)

[PT] Pearce, P. A., Thompson, C. J.: The high density limit for lattice spin models. Commun. Math. Phys. 58, 131-138 (1978)

[R] Ruelle, D.: Thermodynamic formalism. Reading, MA: Addison Wesley 1978

[SV] Schonmann, R. H., Vares, M. E.: The survival of the large dimensional basic contact process. Probab. Th. Rel. Fields 72, 387-393 (1986) 
[Si] Simon, B.: Mean field upper bound on the transition temperature in multicomponent ferromagnets. J. Stat. Phys. 22, 491-493 (1980)

[S1] Slawny, J.: On the mean field theory bound on the magnetization. J. Stat. Phys. 32, 375-388 (1983)

[T1] Thompson, C. J.: Upper bounds for Ising model correlation functions. Commun. Math. Phys. 24, 61-66 (1971)

[T2] Thompson, C. J.: Mathematical statistical mechanics. Princeton, NJ: Princeton University Press (1972)

[T3] Thompson, C. J.: Ising model in the high density limit. Commun. Math. Phys. 36, 255-262 (1974)

[TH] Tasaki, H., Hara, T.: Mean field bound and GHS inequality. J. Stat. Phys. 35, 99-107 (1984)

[V] Vigfusson, J. O.: New upper bounds for the magnetization in ferromagnetic one-component systems. Lett. Math. Phys. 10, 71-77 (1985)

Communicated by M. Aizenman

Received August 8, 1988; in revised form November 18, 1988 
